\title{
On the projective function synchronization of chaos for two gyroscope systems under sinusoidal constraints
}

\author{
Fuhong Min • Albert C. J. Luo
}

Received: 9 April 2013 / Revised: 7 June 2013 / Accepted: 10 June 2013 / Published online: 16 July 2013

(C) Springer-Verlag Berlin Heidelberg 2013

\begin{abstract}
In this paper, the projective function synchronization of chaos between two gyroscope systems with distinct behaviors is investigated under sinusoidal constraints. The objective of the research is to adjust a current gyroscope system to the idealized behaviors in design through synchronization. From the theory of discontinuous dynamical systems, the mechanism of function synchronization of chaotic motions is studied. The analytical conditions for the function synchronization are achieved, and the invariant domain of the function synchronization is obtained. Numerical illustrations for partial and full, projective function synchronization of two gyroscopes with different dynamical behaviors are carried out. The scaling factors in such function synchronization are satisfied through numerical results.
\end{abstract}

Keywords Projective function synchronization .

Chaotic function synchronization •

Synchronization invariant domain - Gyroscope system •

Full function synchronization •

Partial function synchronization

\section{Introduction}

The synchronization of two identical chaotic dynamical systems was extensively investigated since Pecora and Carroll [1] discussed such a topic in 1990. In 1995, Rulkov

\section{F. Min}

School of Electrical and Automation Engineering, Nanjing Normal University, Nanjing 210042, Jiangsu, China

e-mail: minfuhong@njnu.edu.cn

\section{A. C. J. Luo $(\square)$}

Department of Mechanical and Industrial Engineering, Southern Illinois University Edwardsville, Edwardsville, IL 62026-1805, USA

e-mail: aluo@ siue.edu et al. [2] discussed a generalized synchronization of chaos in directionally coupled chaotic systems. In 1999, Mainieri and Rehacek [3] proposed the projective synchronization of the Lorenz systems with the scaling factors. In 2006, Yan et al. [4] discussed the adaptive synchronization control for chaotic symmetric gyroscopes from an adaptive sliding mode control. In 2007, Li [5] discussed the modified projective synchronization of the drive-response systems with a scaling matrix. In 2008, $\mathrm{Hu}$ et al. [6] extended the idea of projective synchronization to hybrid projective synchronization, and the different state variables can be synchronized with different scaling factors. A sliding mode control was used to investigate the generalized projective synchronization of two chaotic gyroscopes with dead-zone input by Hung et al. [7]. In 2010, Chen et al. [8] studied the adaptive generalized synchronization between Chen system and a multi-scroll with uncertain parameters. In 2011, Yu and Li [9] discussed the full state hybrid projective synchronization between different chaotic systems with fully unknown parameters. Wu et al. [10] investigated the generalized function projective synchronization of two hyperchaotic Chen systems for the secure communication schemes. In 2012, Li [11] used the tracking control to investigate the generalized projective synchronization of hyperchaotic systems with unknown parameter and disturbance. Wang [12] utilized the state observer to study a generalized projective synchronization of hyperchaotic systems. Niu et al. [13] investigated the adaptive projective synchronization of different chaotic systems with nonlinearity inputs. In those studies, the Lyapunov method was explored to determine the stability for error systems. The control laws designed are often complicated, and the implementation becomes much difficult in practical engineering. In 2009, Luo [14] developed a different theory for synchronization of dynamical systems under specific constraints from the theory of discontinuous dynamical sys- 
tems. The G-functions were introduced to determine the switchability of a flow from one domain to another in discontinuous dynamical systems. The gyroscope system has a wide spectrum of applications in navigation, aeronautics and space engineering. Min and Luo [15] used such a theory to investigate the chaotic synchronization of two nonlinear gyroscope systems, and Min [16] applied such a new theory to the generalized projective synchronization of a chaotic gyroscope with a periodic gyroscope, and the analytical conditions of synchronization were developed. The noised gyroscope systems should be synchronized with the expected gyroscope systems. In 2013, Min and Luo [17] discussed the parameter characteristics of projective synchronization of two different motion states of the gyroscope system. For security, function synchronization of two dynamical systems should be considered. Thus, the projective function synchronization of two gyroscope systems with different motions under sinusoidal constraints will be of great interest. The investigation objective is to make a current system to synchronize with the expected behaviors in design.

In this paper, from the theory of discontinuous dynamical systems, the necessary and sufficient conditions for the projective function synchronization of two gyroscope systems with different dynamical behaviors will be derived. From the analytical conditions, the invariant domain for the partial and full, projective function synchronizations will be achieved. Finally, numerical illustrations for projective function synchronization of two chaotic gyroscope systems under sinusoidal constraints will be performed.

\section{Problem description}

Consider a gyroscope system as

$\dot{x}_{1}=x_{2}$,

$\dot{x}_{2}=h\left(x_{1}\right)-c_{1} x_{2}-c_{2} x_{2}^{3}+\left(\beta+f_{1} \sin \omega t\right) \sin x_{1}$,

where $x_{1}$ is rotation angle, $x_{2}$ is angular velocity, $f_{1} \sin \omega t$ is a parametric excitation, and a nonlinear function $h\left(x_{1}\right)$ is

$h\left(x_{1}\right)=-\alpha^{2} \frac{\left(1-\cos x_{1}\right)^{2}}{\sin ^{3} x_{1}}$.

The gyroscope system in Eq. (1) is regarded as the master system, and a second controlled gyroscope system is considered as a slave system, i.e.,

$\left\{\begin{array}{l}\dot{y}_{1}=y_{2}-u_{1}(t), \\ \dot{y}_{2}=h\left(y_{1}\right)-c_{1} y_{2}-c_{2} y_{2}^{3}+\left(\beta+f_{2} \sin \omega t\right) \sin y_{1}-u_{2}(t),\end{array}\right.$

with

$h\left(y_{1}\right)=-\frac{\alpha^{2}\left(1-\cos y_{1}\right)^{2}}{\sin ^{3} y_{1}}$.
If parameter $f_{2}$ in Eq. (3) without control is different from $f_{1}$ in Eq. (1), then the master and slave systems have different dynamical behaviors. Consider control laws for the projective functional synchronization with sinusoidal constraint as

$u_{1}=k_{1} \operatorname{sgn}\left(y_{1}-p_{1} \sin x_{1}\right)$,

$u_{2}=k_{2} \operatorname{sgn}\left(y_{2}-p_{2} x_{2} \cos x_{1}\right)$

where $k_{1}$ and $k_{2}$ are controller parameters, $p_{1}$ and $p_{2}$ are scaling factors. Thus the projective function synchronization of the slave system with the master system can be achieved under the sinusoidal constrains. The control laws in Eq. (5) can be designed any forms of functions.

From the foregoing equations, the state variables for the master and slave systems are

$\mathbf{x}=\left(x_{1}, x_{2}\right)^{\mathrm{T}}$ and $\mathbf{y}=\left(y_{1}, y_{2}\right)^{\mathrm{T}}$,

and components for the corresponding vector fields are

$$
\begin{aligned}
& \mathscr{F}_{1}(\mathbf{x}, t)=x_{2} \text { and } \mathscr{F}_{2}(\mathbf{x}, t)=h\left(x_{1}\right)-c_{1} x_{2}-c_{2} x_{2}^{3} \\
& \quad+\left(\beta+f_{1} \sin \omega t\right) \sin x_{1}, \\
& \tilde{F}_{1}(\mathbf{y}, t)=y_{2} \text { and } \tilde{F}_{2}(\mathbf{y}, t)=h\left(y_{1}\right)-c_{1} y_{2}-c_{2} y_{2}^{3} \\
& \quad+\left(\beta+f_{2} \sin \omega t\right) \sin y_{1} .
\end{aligned}
$$

The controlled slave system becomes

$\dot{\mathbf{y}}=\tilde{\mathbf{F}}(\mathbf{y}, t)-\mathbf{u}(\mathbf{x}, \mathbf{y}, t)=\mathbf{F}(\mathbf{y}, t)$,

where the vector field $\mathbf{F}(\mathbf{y}, t)=\left(F_{1}(\mathbf{y}, t), F_{2}(\mathbf{y}, t)\right)^{\mathrm{T}}$.

With the control law in Eq. (5), the controlled gyroscope system will become discontinuous. There are four domains and four boundaries with different vector fields. The four domains $\Omega_{\alpha}(\alpha=1,2,3,4)$ for the controlled slave system are

$$
\begin{aligned}
\Omega_{1}= & \left\{\left(y_{1}, y_{2}\right) \mid y_{1}-p_{1} \sin \left(x_{1}(t)\right)>0, y_{2}\right. \\
& \left.-p_{2} x_{2}(t) \cos \left(x_{1}(t)\right)>0\right\}, \\
\Omega_{2}= & \left\{\left(y_{1}, y_{2}\right) \mid y_{1}-p_{1} \sin \left(x_{1}(t)\right)>0, y_{2}\right. \\
& \left.-p_{2} x_{2}(t) \cos \left(x_{1}(t)\right)<0\right\}, \\
\Omega_{3}= & \left\{\left(y_{1}, y_{2}\right) \mid y_{1}-p_{1} \sin \left(x_{1}(t)\right)<0, y_{2}\right. \\
& \left.-p_{2} x_{2}(t) \cos \left(x_{1}(t)\right)<0\right\}, \\
\Omega_{4}= & \left\{\left(y_{1}, y_{2}\right) \mid y_{1}-p_{1} \sin \left(x_{1}(t)\right)<0, y_{2}\right. \\
& \left.-p_{2} x_{2}(t) \cos \left(x_{1}(t)\right)>0\right\} .
\end{aligned}
$$

The boundaries $\partial \Omega_{\alpha \beta}(\alpha, \beta=1,2,3,4 ; \alpha \neq \beta)$ of four domains are

$$
\begin{aligned}
& \partial \Omega_{12}=\left\{\left(y_{1}, y_{2}\right) \mid \varphi_{12}=y_{2}-p_{2} x_{2}(t) \cos \left(x_{1}(t)\right)=0,\right. \\
&\left.y_{1}-p_{1} \sin \left(x_{1}(t)\right)>0\right\}, \\
& \partial \Omega_{23}=\left\{\left(y_{1}, y_{2}\right) \mid \varphi_{23}=y_{1}-p_{1} \sin \left(x_{1}(t)\right)=0,\right.\left.y_{2}-p_{2} x_{2}(t) \cos \left(x_{1}(t)\right)<0\right\},
\end{aligned}
$$




$$
\begin{aligned}
\partial \Omega_{34}= & \left\{\left(y_{1}, y_{2}\right) \mid \varphi_{34}=y_{2}-p_{2} x_{2}(t) \cos \left(x_{1}(t)\right)=0,\right. \\
& \left.y_{1}-p_{1} \sin \left(x_{1}(t)\right)<0\right\}, \\
\partial \Omega_{41}= & \left\{\left(y_{1}, y_{2}\right) \mid \varphi_{41}=y_{1}-p_{1} \sin \left(x_{1}(t)\right)=0,\right. \\
& \left.y_{2}-p_{2} x_{2}(t) \cos \left(x_{1}(t)\right)>0\right\} .
\end{aligned}
$$

where the subscript $(\cdot)_{\alpha \beta}$ denotes the boundary from $\Omega_{\alpha}$ to $\Omega_{\beta}$. The intersection point of four boundaries $\partial \Omega_{\alpha \beta}(\alpha, \beta=$ $1,2,3,4 ; \alpha \neq \beta$ ) is

$$
\begin{aligned}
\angle \partial \Omega_{\alpha \beta}= & \bigcap_{\alpha=1}^{4} \bigcap_{\beta=1}^{4} \partial \Omega_{\alpha \beta} \\
= & \left\{\left(y_{1}, y_{2}\right) \mid \varphi_{23}=y_{1}-p_{1} \sin \left(x_{1}(t)\right)=0,\right. \\
& \left.y_{2}-p_{2} x_{2}(t) \cos \left(x_{1}(t)\right)=0\right\} .
\end{aligned}
$$

On the domain $\Omega_{\alpha}$, the controlled gyroscope is

$\dot{\mathbf{y}}^{(\alpha)}=\mathbf{F}^{(\alpha)}\left(\mathbf{y}^{(\alpha)}, t\right)$

where

$$
\begin{aligned}
\mathbf{F}^{(\alpha)}\left(\mathbf{y}^{(\alpha)}, t\right)= & \left(F_{1}^{(\alpha)}, F_{2}^{(\alpha)}\right)^{\mathrm{T}}, \\
F_{1}^{(\alpha)}\left(\mathbf{y}^{(\alpha)}, t\right)= & y_{2}^{(\alpha)}-k_{1} \text { for } \alpha=1,2 ; \\
F_{1}^{(\alpha)}\left(\mathbf{y}^{(\alpha)}, t\right)= & y_{2}^{(\alpha)}+k_{1} \text { for } \alpha=3,4 ; \\
F_{2}^{(\alpha)}\left(\mathbf{y}^{(\alpha)}, t\right)= & h\left(y_{1}^{(\alpha)}\right)-c_{1} y_{2}^{(\alpha)}-c_{2}\left(y_{2}^{(\alpha)}\right)^{3} \\
& +\left(\beta+f_{2} \sin \omega t\right) \sin y_{1}^{(\alpha)} \\
& -k_{2} \text { for } \alpha=1,4 ; \\
F_{2}^{(\alpha)}\left(\mathbf{y}^{(\alpha)}, t\right)= & h\left(y_{1}^{(\alpha)}\right)-c_{1} y_{2}^{(\alpha)}-c_{2}\left(y_{2}^{(\alpha)}\right)^{3} \\
& +\left(\beta+f_{2} \sin \omega t\right) \sin y_{1}^{(\alpha)} \\
& +k_{2} \text { for } \alpha=2,3 .
\end{aligned}
$$

and

$h\left(y_{1}^{(\alpha)}\right)=-\alpha^{2}\left(1-\cos y_{1}^{(\alpha)}\right)^{2} / \sin ^{3}\left(y_{1}^{(\alpha)}\right)$.

The dynamical systems on the boundaries $\partial \Omega_{\alpha \beta}$ are

$\dot{\mathbf{y}}^{(\alpha \beta)}=\mathbf{F}^{(\alpha \beta)}\left(\mathbf{y}^{(\alpha \beta)}, \mathbf{x}(t), t\right)$;

$\dot{\mathbf{x}}=\mathscr{F}(\mathbf{x}, t)$

where

$$
\begin{aligned}
& \mathbf{F}^{(\alpha \beta)}=\left(F_{1}^{(\alpha \beta)}, F_{2}^{(\alpha \beta)}\right)^{\mathrm{T}}, \\
& F_{1}^{(\alpha \beta)}\left(\mathbf{y}^{(\alpha \beta)}, t\right)=y_{2}-p_{1} x_{2} \cos x_{1}, \\
& F_{2}^{(\alpha \beta)}\left(\mathbf{y}^{(\alpha \beta)}, t\right)=p_{2}\left(\dot{x}_{2} \cos x_{1}-x_{2}^{2} \sin x_{1}\right) .
\end{aligned}
$$

and

$y_{1}^{(\alpha \beta)}=p_{1} \sin x_{1}$ and $y_{2}^{(\alpha \beta)}=p_{2} x_{2} \cos x_{1}$

on $\partial \Omega_{\alpha \beta}$ for $(\alpha, \beta)=\{(2,3),(4,1)\}$;

$y_{1}^{(\alpha \beta)}=p_{1} \sin x_{1}+C$ and $y_{2}^{(\alpha \beta)}=p_{2} x_{2} \cos x_{1}+C$

on $\partial \Omega_{\alpha \beta}$ for $(\alpha, \beta)=\{(1,2),(3,4)\}$.

From the above equations, the boundaries and corner for the controlled gyroscope system are dependent on time in absolute coordinate. For simplicity, relative coordinates are introduced as

$z_{1}=y_{1}-p_{1} \sin x_{1}$ and $z_{2}=y_{2}-p_{2} x_{2} \cos x_{1}$

The corresponding domains, boundaries and synchronization corner in the relative frame can be expressed by

$$
\begin{aligned}
& \Omega_{1}(t)=\left\{\left(z_{1}, z_{2}\right) \mid z_{1}>0, z_{2}>0\right\}, \\
& \Omega_{2}(t)=\left\{\left(z_{1}, z_{2}\right) \mid z_{1}>0, z_{2}<0\right\}, \\
& \Omega_{3}(t)=\left\{\left(z_{1}, z_{2}\right) \mid z_{1}<0, z_{2}<0\right\}, \\
& \Omega_{4}(t)=\left\{\left(z_{1}, z_{2}\right) \mid z_{1}<0, z_{2}>0\right\} . \\
& \partial \Omega_{12}(t)=\left\{\left(z_{1}, z_{2}\right) \mid \varphi_{12}=z_{2}=0, z_{1}>0\right\}, \\
& \partial \Omega_{23}(t)=\left\{\left(z_{1}, z_{2}\right) \mid \varphi_{23}=z_{1}=0, z_{2}<0\right\}, \\
& \partial \Omega_{34}(t)=\left\{\left(z_{1}, z_{2}\right) \mid \varphi_{34}=z_{2}=0, z_{1}<0\right\}, \\
& \partial \Omega_{41}(t)=\left\{\left(z_{1}, z_{2}\right) \mid \varphi_{41}=z_{1}=0, z_{2}>0\right\} .
\end{aligned}
$$

and

$$
\angle \partial \Omega_{\alpha \beta}=\bigcap_{\alpha=1}^{4} \bigcap_{\beta=1}^{4} \partial \Omega_{\alpha \beta}=\left\{\left(z_{1}, z_{2}\right) \mid z_{1}=0, z_{2}=0\right\} .
$$

The partition of the phase plane in the relative frame is depicted in Fig. 1. The boundaries in the relative coordinates are independent of time. For such domains, boundaries and vertex, the analytical conditions for projective function synchronization of the controlled slave system and master system can be developed from the theory for discontinuous dynamical systems. The controlled gyroscope system in the relative coordinates becomes

$\dot{\mathbf{z}}^{(\alpha)}=\mathbf{g}^{(\alpha)}\left(\mathbf{z}^{(\alpha)}, \mathbf{x}, t\right) \quad$ with $\dot{\mathbf{x}}=\mathscr{F}(\mathbf{x}, t)$

where

$$
\begin{aligned}
\mathbf{g}^{(\alpha)}\left(\mathbf{z}^{(\alpha)}, \mathbf{x}, t\right)= & \left(g_{1}^{(\alpha)}, g_{2}^{(\alpha)}\right)^{\mathrm{T}} ; \\
g_{1}^{(\alpha)}\left(\mathbf{z}^{(\alpha)}, \mathbf{x}, t\right)= & \left.z_{2}^{(\alpha)}+\left(p_{2}-p_{1}\right) x_{2} \cos x_{1}\right) \\
& -k_{1}, \text { for } \alpha=1,2 ; \\
g_{1}^{(\alpha)}\left(\mathbf{z}^{(\alpha)}, \mathbf{x}, t\right)= & \left.z_{2}^{(\alpha)}+\left(p_{2}-p_{1}\right) x_{2} \cos x_{1}\right) \\
& +k_{1}, \text { for } \alpha=3,4 ; \\
g_{2}^{(\alpha)}\left(\mathbf{z}^{(\alpha)}, \mathbf{x}, t\right)= & \Psi\left(\mathbf{z}^{(\alpha)}, \mathbf{x}, t\right)-k_{2}, \text { for } \alpha=1,4 ; \\
g_{2}^{(\alpha)}\left(\mathbf{z}^{(\alpha)}, \mathbf{x}, t\right)= & \Psi\left(\mathbf{z}^{(\alpha)}, \mathbf{x}, t\right)+k_{2}, \text { for } \alpha=2,3 .
\end{aligned}
$$

with

$$
\begin{aligned}
\Psi\left(\mathbf{z}^{(\alpha)}, \mathbf{x}, t\right)= & h\left(z_{1}^{(\alpha)}+p_{1} \sin x_{1}\right)-p_{2} h\left(x_{1}\right) \cos x_{1} \\
& -c_{2}\left[\left(z_{2}^{(\alpha)}+p_{2} x_{2} \cos x_{1}\right)^{3}-p_{2} x_{2}^{3} \cos x_{1}\right] \\
& +x_{2}^{2} \sin x_{1}-c_{1} z_{2}^{(\alpha)}+\beta\left[\sin \left(z_{1}^{(\alpha)}+p_{1} \sin x_{1}\right)\right. \\
& \left.-0.5 p_{2} \sin 2 x_{1}\right]+\left[f_{2} \sin \left(z_{1}^{(\alpha)}+p_{1} \sin x_{1}\right)\right. \\
& \left.-0.5 f_{1} p_{2} \sin 2 x_{1}\right] \times \sin \omega t
\end{aligned}
$$

The equations of motion on the boundary in the relative coordinates become 


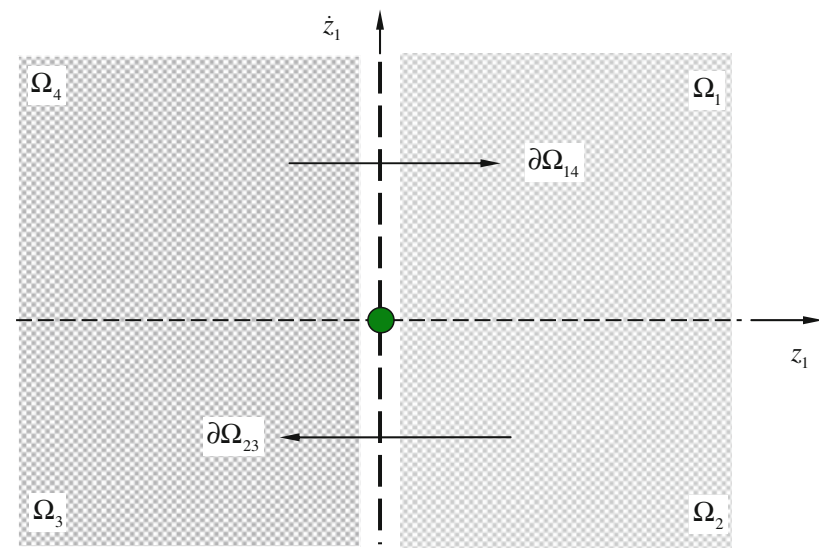

(a)

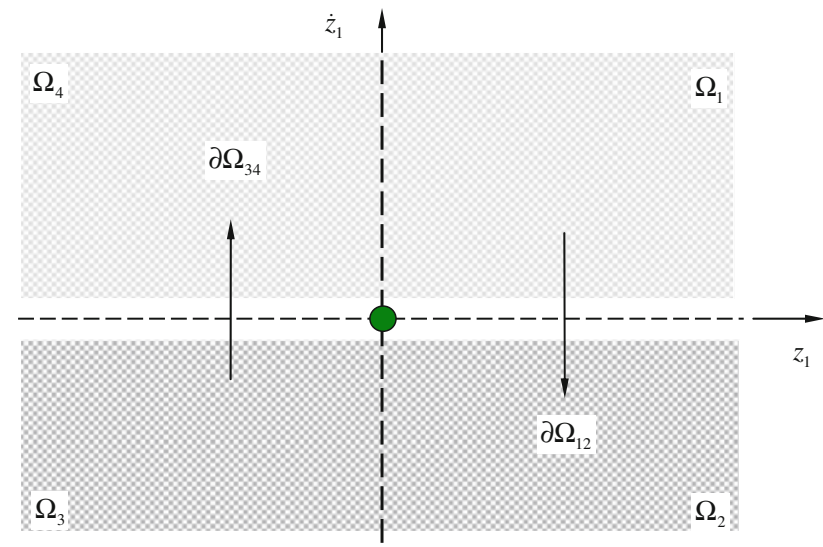

(b)

Fig. 1 Phase plane partitions and boundaries in the relative coordinate. a relative velocity boundary, $\mathbf{b}$ relative displacement boundary

$\dot{\mathbf{z}}^{(\alpha \beta)}=\mathbf{g}^{(\alpha \beta)}\left(\mathbf{z}^{(\alpha \beta)}, \mathbf{x}, t\right) \quad$ with $\dot{\mathbf{x}}=\mathscr{F}(\mathbf{x}, t) ;$

where

$\mathbf{g}^{(\alpha \beta)}\left(\mathbf{z}^{(\alpha \beta)}, \mathbf{x}, t\right)=\left(g_{1}^{(\alpha \beta)}, g_{2}^{(\alpha \beta)}\right)^{\mathrm{T}} ;$

$g_{1}^{(\alpha \beta)}\left(\mathbf{z}^{(\alpha \beta)}, \mathbf{x}, t\right)=z_{2}=0$ and $g_{2}^{(\alpha \beta)}\left(\mathbf{z}^{(\alpha \beta)}, \mathbf{x}, t\right)=0$,

with

$z_{1}^{(\alpha \beta)}=0$ and $z_{2}^{(\alpha \beta)}=0$

on $\partial \Omega_{\alpha \beta}$ for $(\alpha, \beta)=\{(2,3),(4,1)\}$;

$z_{1}^{(\alpha \beta)}=C$ and $z_{2}^{(\alpha \beta)}=0$

on $\partial \Omega_{\alpha \beta}$ for $(\alpha, \beta)=\{(1,2),(3,4)\}$.

\section{Function synchronization}

From the theory of discontinuous dynamical system in Luo $[14,18-20]$, the analytical conditions for function synchronization of two gyroscope systems can be developed. The
G-functions in the relative coordinates are introduced for $z_{m} \in \partial \Omega_{i j}$ at $t=t_{m}$ are introduced as

$$
\begin{aligned}
& G_{\partial \Omega_{i j}}^{(\alpha)}\left(\mathbf{z}_{m}, \mathbf{x}, t_{m \pm}\right) \\
& \quad=\mathbf{n}_{\partial \Omega_{i j}}^{\mathrm{T}} \cdot\left[\mathbf{g}^{(\alpha)}\left(\mathbf{z}_{m}, \mathbf{x}, t_{m \pm}\right)-\mathbf{g}^{(i j)}\left(\mathbf{z}_{m}, \mathbf{x}, t_{m \pm}\right)\right], \\
& G_{\partial \Omega_{i j}}^{(1, \alpha)}\left(\mathbf{z}_{m}, \mathbf{x}, t_{m \pm}\right) \\
& \quad=\mathbf{n}_{\partial \Omega_{i j}}^{\mathrm{T}} \cdot\left[D \mathbf{g}^{(\alpha)}\left(\mathbf{z}_{m}, \mathbf{x}, t_{m \pm}\right)-D \mathbf{g}^{(i j)}\left(\mathbf{z}_{m}, \mathbf{x}, t_{m \pm}\right)\right] .
\end{aligned}
$$

where $G_{\partial \Omega_{i j}}^{(\alpha)}\left(\mathbf{z}_{m}, \mathbf{x}, t_{m \pm}\right)$ and $G_{\partial \Omega_{i j}}^{(1, \alpha)}\left(\mathbf{z}_{m}, \mathbf{x}, t_{m \pm}\right)$ are the zeroorder and first-order G-functions of the flow in $\Omega_{\alpha}(\alpha \in$ $\{i, j\})$ at the boundary $\partial \Omega_{i j}(i, j=1,2,3,4)$. The switching $t_{m}$ represents the time of the motion on the boundary and the switching time $t_{m \pm}=t_{m} \pm 0$ means motions in domains very close to the boundary rather than on the boundary. The normal vectors of the relative boundaries in Eq. (20) are

$\mathbf{n}_{\partial \Omega_{12}}=\mathbf{n}_{\partial \Omega_{34}}=(0,1)^{\mathrm{T}}$ and $\mathbf{n}_{\partial \Omega_{23}}=\mathbf{n}_{\partial \Omega_{14}}=(1,0)^{\mathrm{T}}$.

The corresponding G-functions at boundary $\partial \Omega_{i j}(i, j=$ 1, 2,3,4) from Eqs. (22)-(24) are for domain $\Omega_{\alpha}(\alpha \in$ $\{1,2,3,4\})$

$G_{\partial \Omega_{12}}^{(\alpha)}\left(\mathbf{z}_{m}, \mathbf{x}, t_{m \pm}\right)=G_{\partial \Omega_{34}}^{(\alpha)}\left(\mathbf{z}_{m}, \mathbf{x}, t_{m \pm}\right)=g_{2}^{(\alpha)}\left(\mathbf{z}_{m}, \mathbf{x}, t_{m \pm}\right)$,
$G_{\partial \Omega_{23}}^{(\alpha)}\left(\mathbf{z}_{m}, \mathbf{x}, t_{m \pm}\right)=G_{\partial \Omega_{14}}^{(\alpha)}\left(\mathbf{z}_{m}, \mathbf{x}, t_{m \pm}\right)=g_{1}^{(\alpha)}\left(\mathbf{z}_{m}, \mathbf{x}, t_{m \pm}\right) ;$
$G_{\partial \Omega_{12}}^{(1, \alpha)}\left(\mathbf{z}_{m}, \mathbf{x}, t_{m \pm}\right)=G_{\partial \Omega_{34}}^{(1, \alpha)}\left(\mathbf{z}_{m}, \mathbf{x}, t_{m \pm}\right)=D g_{2}^{(\alpha)}\left(\mathbf{z}_{m}, \mathbf{x}, t_{m \pm}\right)$,
$G_{\partial \Omega_{23}}^{(1, \alpha)}\left(\mathbf{z}_{m}, \mathbf{x}, t_{m \pm}\right)=G_{\partial \Omega_{14}}^{(1, \alpha)}\left(\mathbf{z}_{m}, \mathbf{x}, t_{m \pm}\right)=D g_{1}^{(\alpha)}\left(\mathbf{z}_{m}, \mathbf{x}, t_{m \pm}\right) ;$

where

$$
\begin{aligned}
D g_{1}^{(\alpha)}\left(\mathbf{z}^{(\alpha)}, \mathbf{x}, t\right) & \\
= & h\left(z_{1}^{(\alpha)}+p_{1} \sin x_{1}\right)-p_{1} h\left(x_{1}\right) \cos x_{1} \\
& -c_{1}\left[z_{2}^{(\alpha)}+\left(p_{2}-p_{1}\right) x_{2} \cos x_{1}\right] \\
& -c_{2}\left[\left(z_{2}^{(\alpha)}+p_{2} x_{2} \cos x_{1}\right)^{3}-p_{1} x_{2}^{3} \cos x_{1}\right] \\
& +\left(\beta+f_{2} \sin \omega t\right) \sin \left(z_{1}^{(\alpha)}+p_{1} \sin x_{1}\right) \\
& -\frac{1}{2} p_{1}\left(\beta+f_{1} \sin \omega t\right) \sin 2 x_{1}+p_{1} x_{2} \sin x_{1}, \\
D g_{2}^{(\alpha)}\left(\mathbf{z}^{(\alpha)}, \mathbf{x}, t\right) & =\left[h_{1}\left(z_{1}^{(\alpha)}+p_{1} \sin x_{1}\right)+\left(\beta+f_{2} \sin \omega t\right)\right. \\
\quad & \left.\times \cos \left(z_{1}^{(\alpha)}+p_{1} \sin x_{1}\right)\right] F_{1}\left(\mathbf{z}^{(\alpha)}+\mathbf{x}, t\right) \\
& -\left[c_{1}+3 c_{2}\left(z_{2}^{(\alpha)}+p_{2} x_{2} \cos x_{1}\right)^{2}\right] \\
& \times F_{2}\left(\mathbf{z}^{(\alpha)}+\mathbf{x}, t\right)+\left[f_{2} \sin \left(z_{1}^{(\alpha)}+p_{1} \sin x_{1}\right)\right. \\
& \left.-\frac{1}{2} p_{2} f_{1} \sin 2 x_{1}\right]
\end{aligned}
$$




$$
\begin{aligned}
& \times \omega \cos \omega t-p_{2}\left[h_{1}\left(x_{1}\right) \cos x_{1}\right. \\
& \left.+\left(\beta+f_{1} \sin \omega t\right) \cos ^{2} x_{1}\right] \\
& \times F_{1}(\mathbf{x}, t)+p_{2}\left(c_{1} \cos x_{1}\right. \\
& \left.+3 c_{2} x_{2}^{2} \cos x_{1}+3 x_{2} \sin x_{1}\right) \\
& \times F_{2}(\mathbf{x}, t) ;
\end{aligned}
$$

with

$$
\begin{aligned}
h_{1}\left(z_{1}^{(\alpha)}+p_{1} \sin x_{1}\right) & =\alpha^{2}\left(\cos ^{3}\left(z_{1}^{(\alpha)}+p_{1} \sin x_{1}\right)\right. \\
& -4 \cos ^{2}\left(z_{1}^{(\alpha)}+p_{1} \sin x_{1}\right) \\
& \left.+5 \cos \left(z_{1}^{(\alpha)}+p_{1} \sin x_{1}\right)-2\right) / \\
& \sin ^{4}\left(z_{1}^{(\alpha)}+p_{1} \sin x_{1}\right) \\
h_{1}\left(x_{1}\right) & =\alpha^{2}\left(\cos ^{3} x_{1}-4 \cos ^{2} x_{1}\right. \\
& \left.+5 \cos x_{1}-2\right) / \sin ^{4} x_{1} .
\end{aligned}
$$

for $\alpha=1,2,3,4$.

The G-functions in domains $\Omega_{\alpha}(\alpha \in\{1,2,3,4\})$ with respect to the boundary are

$$
\begin{aligned}
& G_{\partial \Omega_{12}}^{(\alpha)}\left(\mathbf{z}^{(\alpha)}, \mathbf{x}, t\right)=G_{\partial \Omega_{34}}^{(\alpha)}\left(\mathbf{z}^{(\alpha)}, \mathbf{x}, t\right)=g_{2}^{(\alpha)}\left(\mathbf{z}^{(\alpha)}, \mathbf{x}, t\right), \\
& G_{\partial \Omega_{23}}^{(\alpha)}\left(\mathbf{z}^{(\alpha)}, \mathbf{x}, t\right)=G_{\partial \Omega_{14}}^{(\alpha)}\left(\mathbf{z}^{(\alpha)}, \mathbf{x}, t\right)=g_{1}^{(\alpha)}\left(\mathbf{z}^{(\alpha)}, \mathbf{x}, t\right) ; \\
& G_{\partial \Omega_{12}}^{(1, \alpha)}\left(\mathbf{z}^{(\alpha)}, \mathbf{x}, t\right)=G_{\partial \Omega_{34}}^{(1, \alpha)}\left(\mathbf{z}^{(\alpha)}, \mathbf{x}, t\right)=D g_{2}^{(\alpha)}\left(\mathbf{z}^{(\alpha)}, \mathbf{x}, t\right), \\
& G_{\partial \Omega_{23}}^{(1, \alpha)}\left(\mathbf{z}^{(\alpha)}, \mathbf{x}, t\right)=G_{\partial \Omega_{14}}^{(1, \alpha)}\left(\mathbf{z}^{(\alpha)}, \mathbf{x}, t\right)=D g_{1}^{(\alpha)}\left(\mathbf{z}^{(\alpha)}, \mathbf{x}, t\right) .
\end{aligned}
$$

From the theory for discontinuous dynamical systems [19, 20], the switchability of a flow to the boundary $\partial \Omega_{\alpha \beta}$ for $(\alpha, \beta)=\{(1,2),(2,3),(3,4),(1,4)\}$. The sliding flow of the controlled gyroscope system on the separation boundary, the conditions for projective function synchronization of the two gyroscope systems at the intersection point are

$$
\left.\begin{array}{l}
G_{\partial \Omega_{14}}^{(1)}\left(\mathbf{z}_{m}, \mathbf{x}, t_{m-}\right)=g_{1}^{(1)}\left(\mathbf{z}_{m}, \mathbf{x}, t_{m-}\right)<0, \\
G_{\partial \Omega_{12}}^{(1)}\left(\mathbf{z}_{m}, \mathbf{x}, t_{m-}\right)=g_{2}^{(1)}\left(\mathbf{z}_{m}, \mathbf{x}, t_{m-}\right)<0,
\end{array}\right\}
$$$$
\text { for } \mathbf{z}_{m} \in \partial \Omega_{12} \cap \partial \Omega_{14} \text { on } \Omega_{1} \text {; }
$$$$
\left.\begin{array}{l}
G_{\partial \Omega_{12}}^{(2)}\left(\mathbf{z}_{m}, \mathbf{x}, t_{m-}\right)=g_{2}^{(2)}\left(\mathbf{z}_{m}, \mathbf{x}, t_{m-}\right)>0, \\
G_{\partial \Omega_{23}}^{(2)}\left(\mathbf{z}_{m}, \mathbf{x}, t_{m-}\right)=g_{1}^{(2)}\left(\mathbf{z}_{m}, \mathbf{x}, t_{m-}\right)<0,
\end{array}\right\}
$$$$
\text { for } \mathbf{z}_{m} \in \partial \Omega_{12} \cap \partial \Omega_{23} \text { on } \Omega_{2} \text {; }
$$

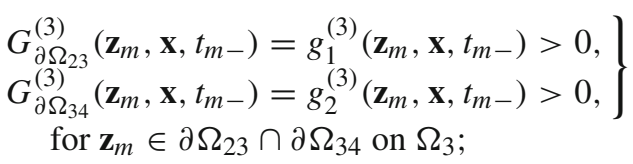

$$
\begin{aligned}
& G_{\partial \Omega_{34}}^{(4)}\left(\mathbf{z}_{m}, \mathbf{x}, t_{m-}\right)=g_{2}^{(4)}\left(\mathbf{z}_{m}, \mathbf{x}, t_{m-}\right)<0, \\
& \left.G_{\partial \Omega_{14}}^{(4)}\left(\mathbf{z}_{m}, \mathbf{x}, t_{m-}\right)=g_{1}^{(4)}\left(\mathbf{z}_{m}, \mathbf{x}, t_{m-}\right)>0,\right\} \\
& \text { for } \mathbf{z}_{m} \in \partial \Omega_{34} \cap \partial \Omega_{14} \text { on } \Omega_{4} \text {. }
\end{aligned}
$$

From Eq. (23), the following functions are defined as

$$
\begin{aligned}
& g_{1}\left(\mathbf{z}^{(\alpha)}, \mathbf{x}, t\right) \equiv g_{1}^{(\alpha)}\left(\mathbf{z}^{(\alpha)}, \mathbf{x}, t\right) \\
& \quad=z_{2}^{(\alpha)}+\left(p_{2}-p_{1}\right) x_{2} \cos x_{1}-k_{1} \\
& \quad \text { in } \Omega_{\alpha} \text { for } \alpha=1,2 ; \\
& g_{2}\left(\mathbf{z}^{(\alpha)}, \mathbf{x}, t\right) \equiv g_{1}^{(\alpha)}\left(\mathbf{z}^{(\alpha)}, \mathbf{x}, t\right) \\
& \quad=z_{2}^{(\alpha)}+\left(p_{2}-p_{1}\right) x_{2} \cos x_{1}+k_{1} \\
& \quad \text { in } \Omega_{\alpha} \text { for } \alpha=3,4 \\
& g_{3}\left(\mathbf{z}^{(\alpha)}, \mathbf{x}, t\right) \equiv g_{2}^{(\alpha)}\left(\mathbf{z}^{(\alpha)}, \mathbf{x}, t\right) \\
& \quad=\psi\left(\mathbf{z}^{(\alpha)}, \mathbf{x}, t\right)-k_{2} \\
& \quad \text { in } \Omega_{\alpha} \text { for } \alpha=1,4 \\
& g_{4}\left(\mathbf{z}^{(\alpha)}, \mathbf{x}, t\right) \equiv g_{2}^{(\alpha)}\left(\mathbf{z}^{(\alpha)}, \mathbf{x}, t\right) \\
& \quad=\psi\left(\mathbf{z}^{(\alpha)}, \mathbf{x}, t\right)+k_{2} \\
& \quad \text { in } \Omega_{\alpha} \text { for } \alpha=2,3 .
\end{aligned}
$$

Therefore, the synchronization conditions in Eqs. (35) become

$$
\begin{aligned}
& g_{1}\left(\mathbf{z}_{m}, \mathbf{x}, t_{m-}\right)=z_{2 m}+\left(p_{2}-p_{1}\right) x_{2} \cos x_{1}-k_{1}<0 \\
& g_{2}\left(\mathbf{z}_{m}, \mathbf{x}, t_{m-}\right)=z_{2 m}+\left(p_{2}-p_{1}\right) x_{2} \cos x_{1}+k_{1}>0 \\
& g_{3}\left(\mathbf{z}_{m}, \mathbf{x}, t_{m-}\right)=\psi\left(\mathbf{z}_{m}, \mathbf{x}, t_{m-}\right)-k_{2}<0 \\
& g_{4}\left(\mathbf{z}_{m}, \mathbf{x}, t_{m-}\right)=\psi\left(\mathbf{z}_{m}, \mathbf{x}, t_{m-}\right)+k_{2}>0
\end{aligned}
$$

Setting $\mathbf{z}_{m}=\mathbf{0}$, the analytical conditions of projective function synchronization for the controlled gyroscope with the expected chaotic gyroscope system are

$$
\begin{aligned}
& g_{1}\left(\mathbf{z}_{m}, \mathbf{x}, t_{m-}\right)=\left(p_{2}-p_{1}\right) x_{2} \cos x_{1}-k_{1}<0, \\
& g_{2}\left(\mathbf{z}_{m}, \mathbf{x}, t_{m-}\right)=\left(p_{2}-p_{1}\right) x_{2} \cos x_{1}+k_{1}>0, \\
& g_{3}\left(\mathbf{z}_{m}, \mathbf{x}, t_{m-}\right)=\psi\left(\mathbf{x}, t_{m-}\right)-k_{2}<0, \\
& g_{4}\left(\mathbf{z}_{m}, \mathbf{x}, t_{m-}\right)=\psi\left(\mathbf{x}, t_{m-}\right)+k_{2}>0 .
\end{aligned}
$$

where

$$
\begin{aligned}
& \psi\left(\mathbf{x}, t_{m-}\right) \\
&= x_{2}^{2} \sin x_{1}+h\left(p_{1} \sin x_{1}\right)-p_{2} h\left(x_{1}\right) \cos x_{1} \\
&-c_{2}\left[\left(p_{2} x_{2} \cos x_{1}\right)^{3}-p_{2} x_{2}^{3} \cos x_{1}\right] \\
&+\beta\left[\sin \left(p_{1} \sin x_{1}\right)-0.5 p_{2} \sin 2 x_{1}\right] \\
&+\left[f_{2} \sin \left(p_{1} \sin x_{1}\right)-0.5 f_{1} p_{2} \sin 2 x_{1}\right] \sin \omega t_{m-}
\end{aligned}
$$

and the synchronization invariant domain is

$$
\begin{aligned}
& -k_{1}<\left(p_{2}-p_{1}\right) x_{2} \cos x_{1}<k_{1} \\
& -k_{2}<\Psi\left(\mathbf{x}, t_{m-}\right)<k_{2}
\end{aligned}
$$

from which the synchronization invariant domain depends on the master system, control parameters $k_{1}, k_{2}$, and scaling factors $p_{1}, p_{2}$. From Luo $[19,20]$, if the projective function synchronization for two gyroscope systems disappears, the vanishing conditions for projective function synchronization on $\partial \Omega_{\alpha \beta}$ for $(\alpha, \beta)=\{(1,4),(2,3)\}$ are 

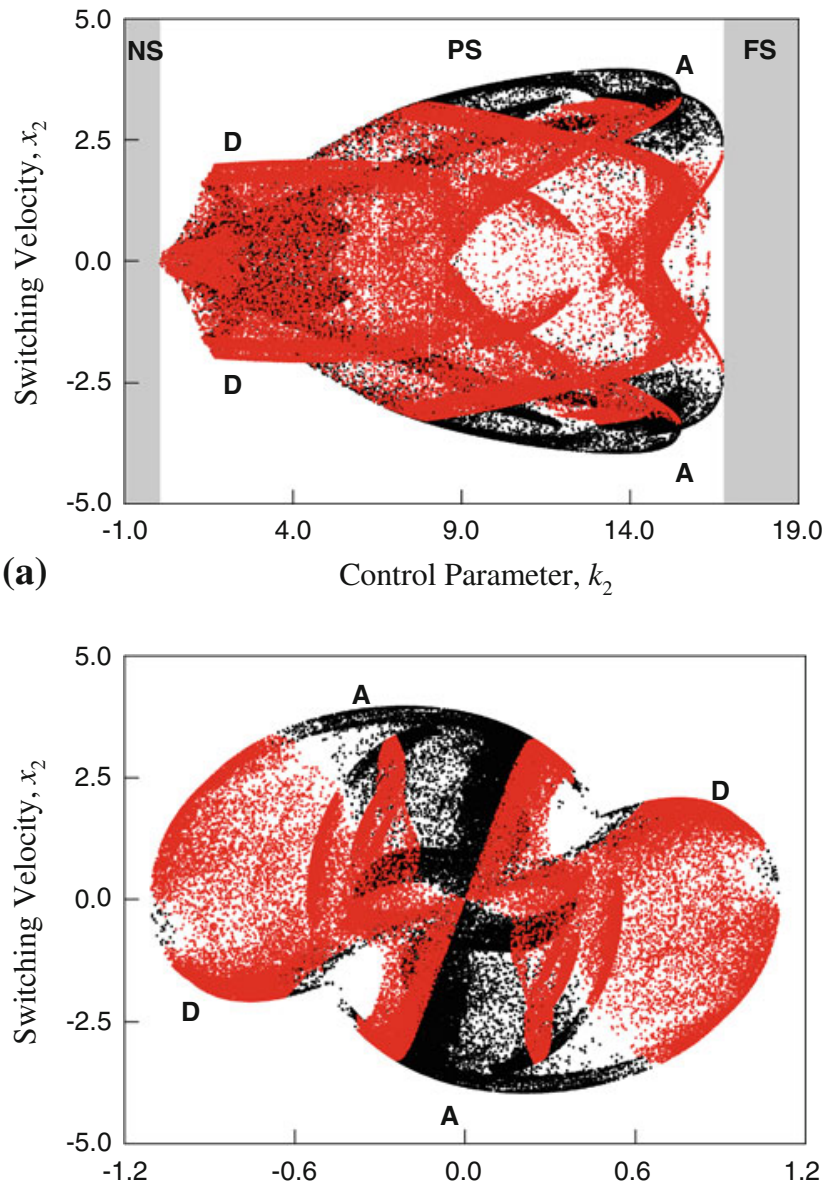

(c)

Switching Displacement, $x_{1}$

Fig. 2 The projective function synchronization scenario for the switching points against the control parameter $k_{2}$. Master system: a switching velocity, b switching phase, c switching points in phase. Slave system:

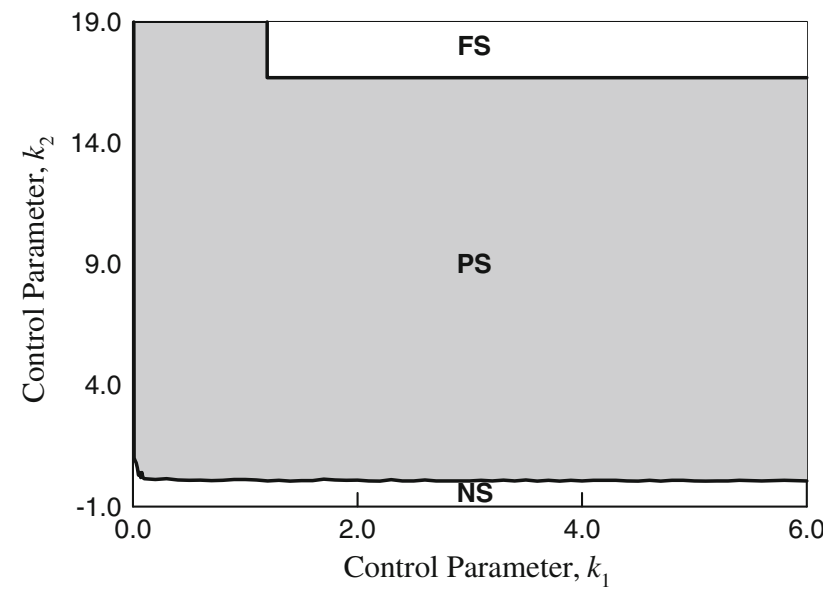

Fig. 3 The parameter map $\left(k_{1}, k_{2}\right)$ for projective function synchronicity of the controlled periodic gyroscope with the chaotic gyroscope system (FS full synchronization, $P S$ partial synchronization, $N S$ nonsynchronization)
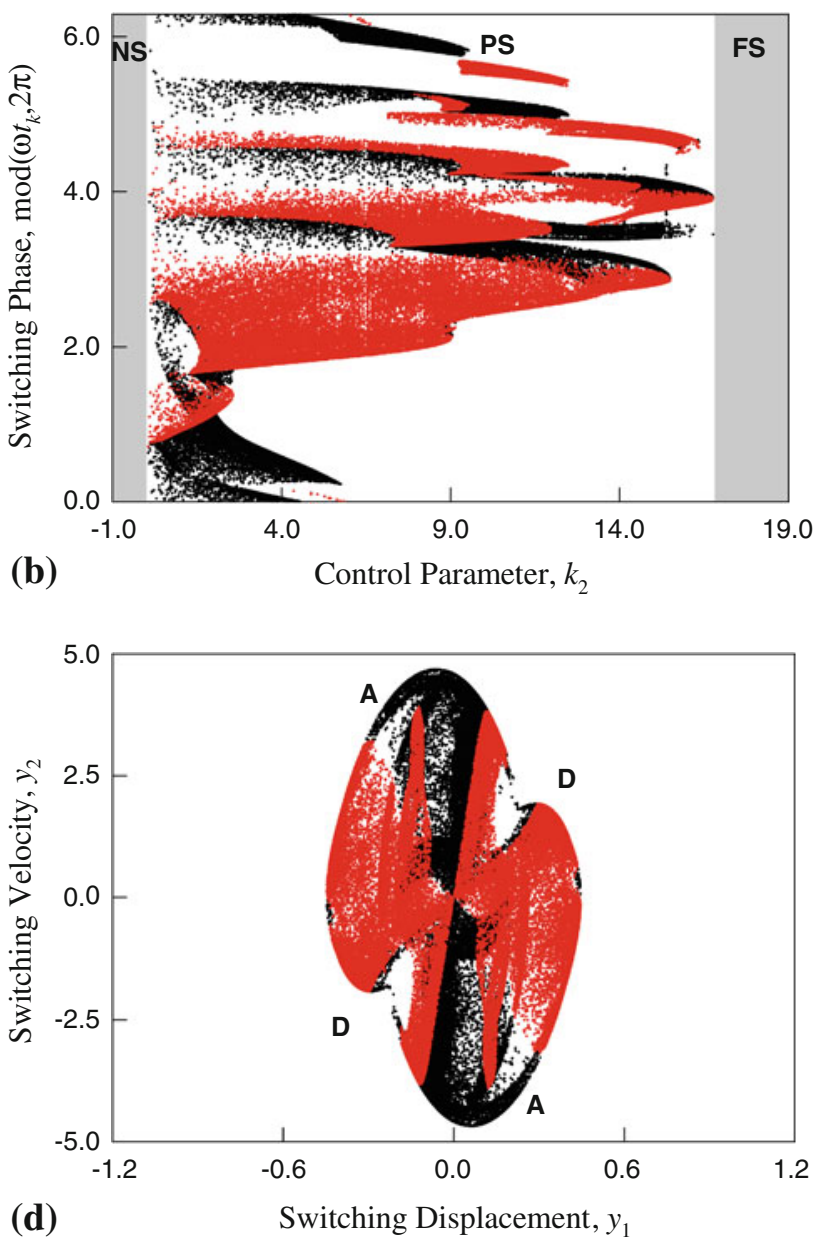

d switching points in phase space (FS full synchronization, PS partial synchronization, NS non-synchronization, A synchronization appearance, D synchronization disappearance)

$$
\left.\begin{array}{l}
g_{1}\left(\mathbf{z}_{m}^{(\alpha)}, \mathbf{x}, t_{m \mp}\right)=z_{2 m}^{(\alpha)}-k_{1}=0, \\
D g_{1}\left(\mathbf{z}_{m}^{(\alpha)}, \mathbf{x}, t_{m \mp}\right)=\mathscr{G}\left(\mathbf{z}_{m}^{(\alpha)}, \mathbf{x}, t_{m \mp}\right)>0, \\
g_{2}\left(\mathbf{z}_{m}^{(\beta)}, \mathbf{x}, t_{m-}\right)=z_{2 m}^{(\beta)}+k_{1}>0,
\end{array}\right\}
$$

and the vanishing conditions of projective function synchronization on another boundary $\partial \Omega_{\alpha \beta}$ for $(\alpha, \beta) \in$ $\{(1,2),(4,3)\}$ are

$$
\left.\begin{array}{l}
g_{3}\left(\mathbf{z}_{m}^{(\alpha)}, \mathbf{x}, t_{m \mp}\right)=\mathscr{G}\left(\mathbf{z}_{m}^{(\alpha)}, \mathbf{x}, t_{m \mp}\right)-k_{2}=0, \\
D g_{3}\left(\mathbf{z}_{m}^{(\alpha)}, \mathbf{x}, t_{m \mp}\right)=D \mathscr{G}\left(\mathbf{z}_{m}^{(\alpha)}, \mathbf{x}, t_{m \mp}\right)>0, \\
g_{4}\left(\mathbf{z}_{m}^{(\beta)}, \mathbf{x}, t_{m-}\right)=\mathscr{G}\left(\mathbf{z}_{m}^{(\beta)}, \mathbf{x}, t_{m-}\right)+k_{2}>0,
\end{array}\right\}
$$

for $\dot{z}_{m+\varepsilon}=y_{2}-p_{2} x_{2} \cos x_{1}>0$, and 


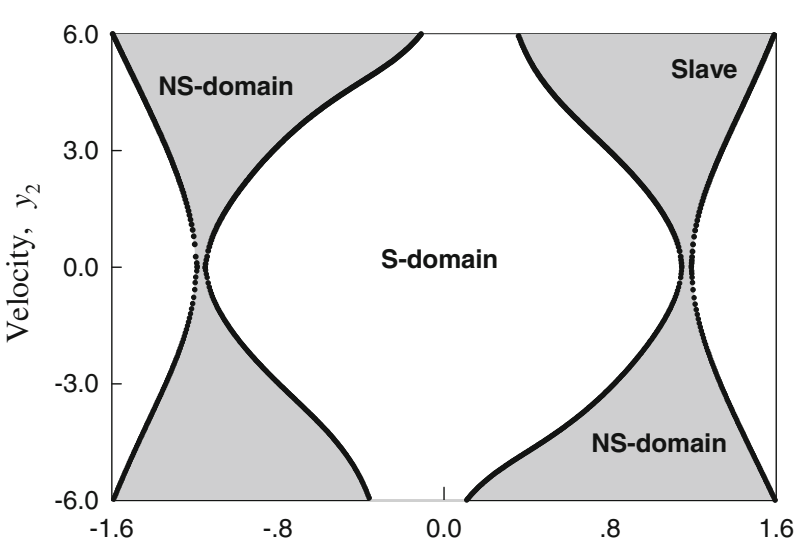

(a)

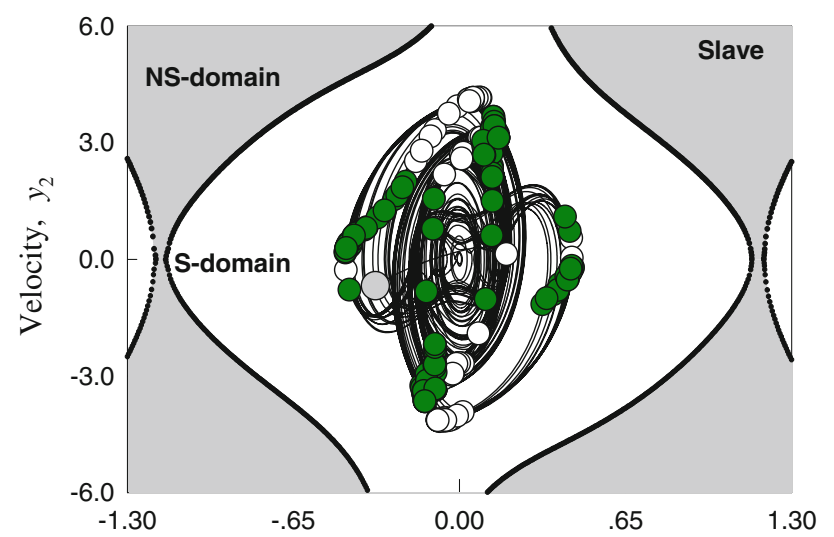

(c)

Displacement, $y_{1}$

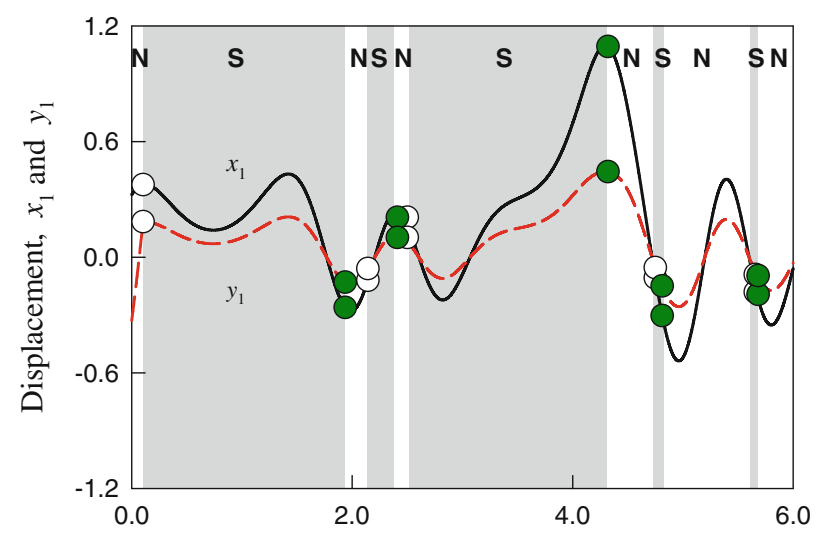

(e)

Time, $t$

Fig. 4 The partial function synchronization for the periodic motion with the chaotic attractor of gyroscope dynamical systems: a function synchronization invariant domain, $\mathbf{b}$ trajectories of master system, c trajectories of slave system, and $\mathbf{d}$ velocity $y_{2}$ versus $x_{2} \cos x_{1}$, e

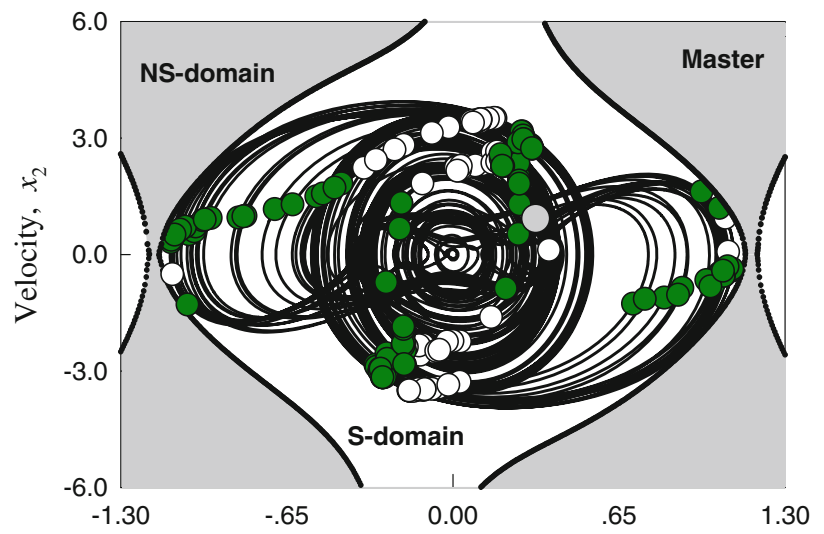

(b)

Displacement, $x_{1}$

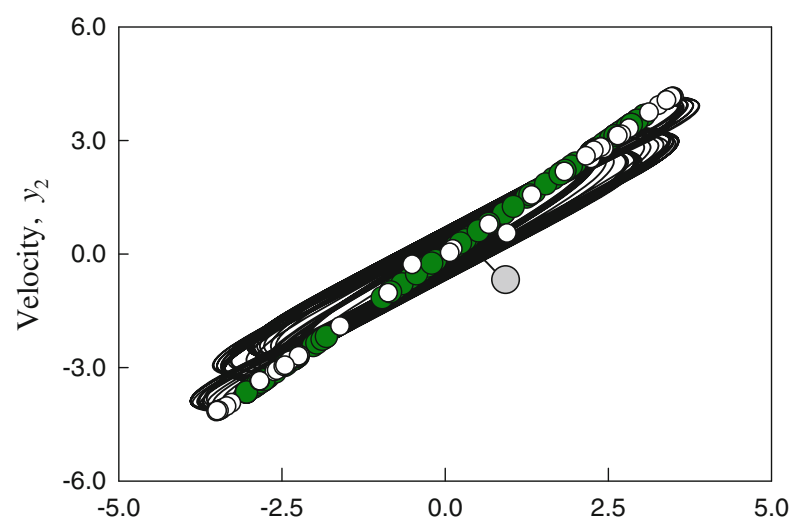

(d)

$x_{2} \cos x_{1}$

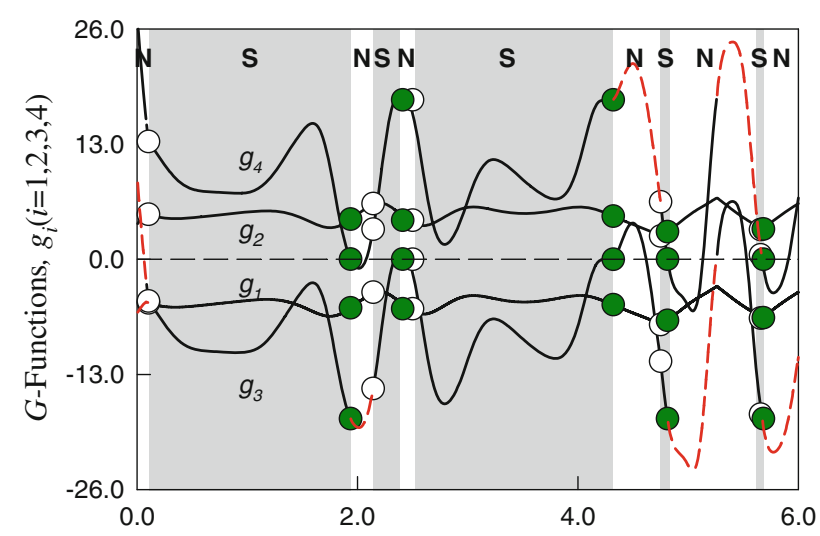

(f)

Time $t$

displacement responses, f G-function responses. (S synchronization, N non-synchronization. Hollow circular symbols synchronization appearance, filled circular symbols synchronization disappearance)

for $\dot{z}_{m+\varepsilon}=y_{2}-p_{2} x_{2} \cos x_{1}<0$.

From Luo $[19,20]$, the onset conditions of projective function synchronization on $\partial \Omega_{\alpha \beta}$ for $(\alpha, \beta) \in\{(1,4),(2,3)\}$ are 


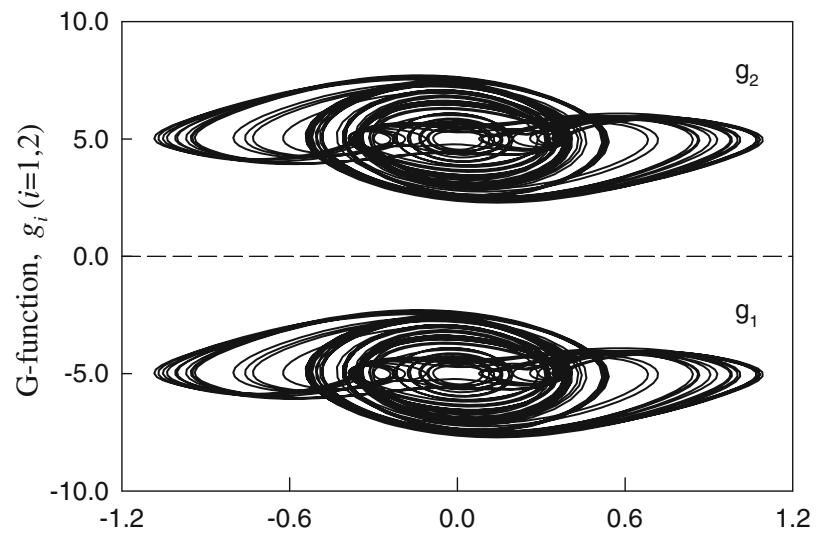

(a)

Displacement, $x_{1}$

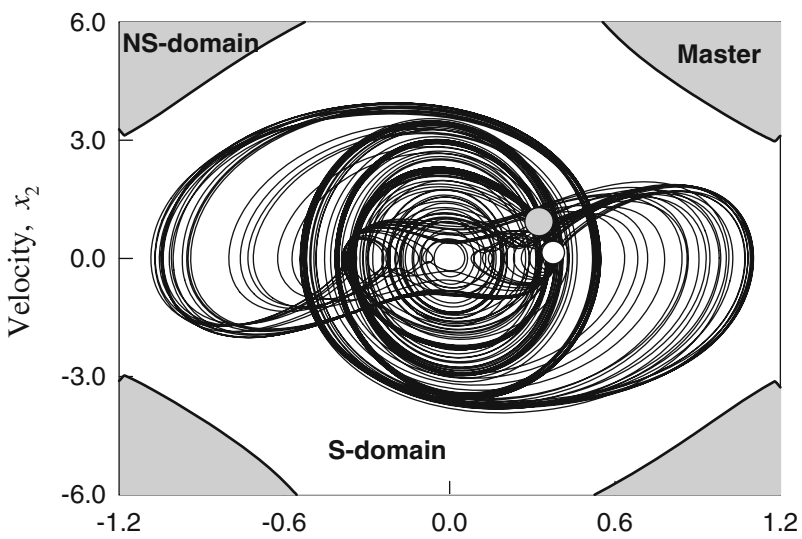

(c)

Displacement, $x_{1}$

Fig. 5 The full generalized synchronization in the invariant domain: a G-function $g_{i}(i=1,2)$ distribution, b G-function $g_{i}(i=3,4)$ distribution, $\mathbf{c}$ trajectories of master system, $\mathbf{d}$ trajectories of slave sys-

$$
\left.\begin{array}{l}
g_{1}\left(\mathbf{z}_{m}^{(\alpha)}, \mathbf{x}, t_{m \pm}\right)=z_{2 m}^{(\alpha)}-k_{1}=0 \\
D g_{1}\left(\mathbf{z}_{m}^{(\alpha)}, \mathbf{x}, t_{m \pm}\right)=\mathscr{G}\left(\mathbf{z}_{m}^{(\alpha)}, \mathbf{x}, t_{m \pm}\right)>0 \\
g_{2}\left(\mathbf{z}_{m}^{(\beta)}, \mathbf{x}, t_{m-}\right)=z_{2 m}^{(\beta)}+k_{1}>0
\end{array}\right\}
$$

for $z_{m-\varepsilon}=y_{1}-p_{1} \sin x_{1}>0$, and

$$
\left.\begin{array}{l}
g_{1}\left(\mathbf{z}_{m}^{(\alpha)}, \mathbf{x}, t_{m-}\right)=z_{2 m}^{(\alpha)}-k_{1}<0 \\
g_{2}\left(\mathbf{z}_{m}^{(\beta)}, \mathbf{x}, t_{m \pm}\right)=z_{2 m}^{(\beta)}+k_{1}=0, \\
D g_{2}\left(\mathbf{z}_{m}^{(\beta)}, \mathbf{x}, t_{m \pm}\right)=\mathscr{G}\left(\mathbf{z}_{m}^{(\beta)}, \mathbf{x}, t_{m \pm}\right)<0
\end{array}\right\}
$$

for $z_{m-\varepsilon}=y_{1}-p_{1} \sin x_{1}>0$.

and the onset conditions of projective function synchronization on another boundary $\partial \Omega_{\alpha \beta}$ for $(\alpha, \beta)=\{(1,2),(4,3)\}$ are

$$
\left.\begin{array}{l}
g_{3}\left(\mathbf{z}_{m}^{(\alpha)}, \mathbf{x}, t_{m \pm}\right)=\mathscr{G}\left(\mathbf{z}_{m}^{(\alpha)}, \mathbf{x}, t_{m \pm}\right)-k_{2}=0, \\
D g_{3}\left(\mathbf{z}_{m}^{(\alpha)}, \mathbf{x}, t_{m \pm}\right)=D \mathscr{G}\left(\mathbf{z}_{m}^{(\alpha)}, \mathbf{x}, t_{m \pm}\right)>0 ; \\
g_{4}\left(\mathbf{z}_{m}^{(\beta)}, \mathbf{x}, t_{m-}\right)=\mathscr{G}\left(\mathbf{z}_{m}^{(\beta)}, \mathbf{x}, t_{m-}\right)+k_{2}>0
\end{array}\right\}
$$

for $\dot{z}_{m-\varepsilon}=y_{2}-p_{2} x_{2} \cos x_{1}>0$, and

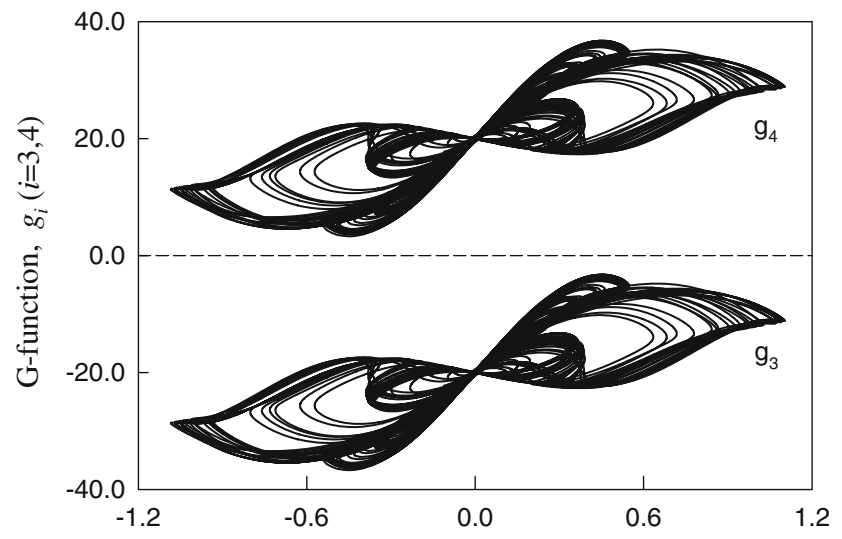

(b)

Displacement, $x_{1}$

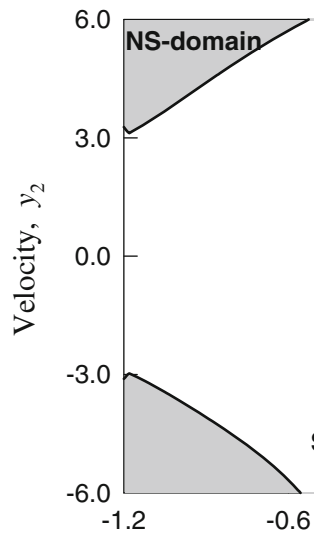

(d)
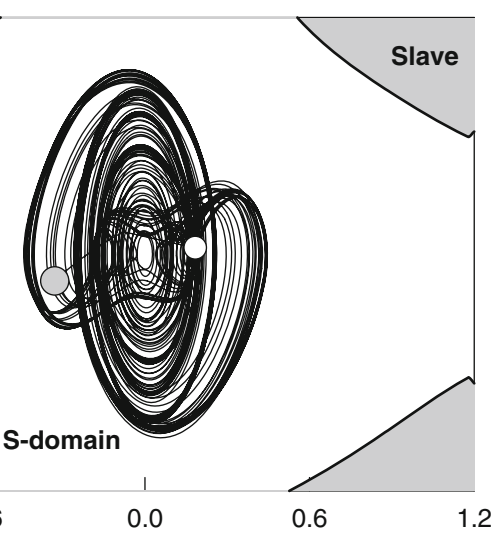

Displacement, $y_{1}$

tem. $S$ synchronization, NS non-synchronization. Hollow circular symbols synchronization appearance, filled circular symbols synchronization disappearance)

$$
\left.\begin{array}{l}
g_{3}\left(\mathbf{z}_{m}^{(\alpha)}, \mathbf{x}, t_{m-}\right)=\mathscr{G}\left(\mathbf{z}_{m}^{(\alpha)}, \mathbf{x}, t_{m-}\right)-k_{2}<0 \\
g_{4}\left(\mathbf{z}_{m}^{(\beta)}, \mathbf{x}, t_{m \pm}\right)=\mathscr{G}\left(\mathbf{z}_{m}^{(\beta)}, \mathbf{x}, t_{m \pm}\right)+k_{2}=0 \\
D g_{4}\left(\mathbf{z}_{m}^{(\beta)}, \mathbf{x}, t_{m \pm}\right)=D \mathscr{G}\left(\mathbf{z}_{m}^{(\beta)}, \mathbf{x}, t_{m \pm}\right)<0
\end{array}\right\}
$$

for $\dot{z}_{m-\varepsilon}=y_{2}-p_{2} x_{2} \cos x_{1}<0$.

\section{Numerical illustrations}

Numerical results will be presented to make a better understanding of the projective function synchronization of two gyroscope systems with different dynamical behaviors. The parameters of nonlinear gyroscope systems are considered as

$$
\begin{aligned}
& \alpha^{2}=100, \beta=1, c_{1}=0.5, c_{2}=0.05, \omega=2, \\
& f_{1}=38.0, f_{2}=35.9, p_{1}=0.5, p_{2}=1.2 \\
& \left(x_{1}, x_{2}\right)=(0.17391,1.36406) \text { and } \\
& \left(y_{1}, y_{2}\right)=(0.32878,0.67165) .
\end{aligned}
$$

With the above parameters and initial conditions, the master system possesses chaotic motion but the slave system without control possesses a period-4 motion. 


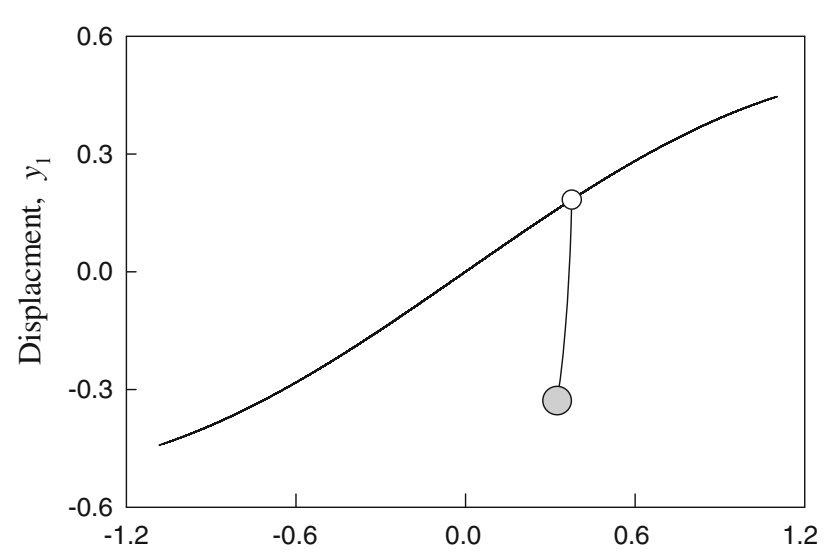

(a)

Displacement, $x_{1}$

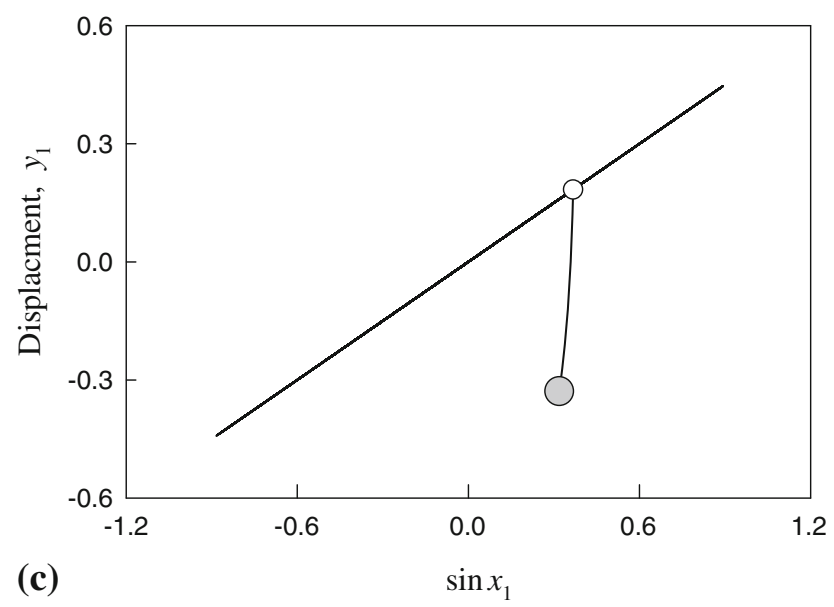

Fig. 6 The relationship of state variables of two coupled gyroscopes with full function synchronization: a the displacement $x_{1}$ versus $y_{1}, \mathbf{b}$ the velocity $x_{2}$ versus $y_{2}, \mathbf{c}$ the displacement $y_{1}$ versus $\sin x_{1}$, and $\mathbf{d}$ the

From the analytical conditions for synchronization of two gyroscope systems, the synchronization switching scenario of the controlled gyroscope system varying with control parameter $k_{2}$ is plotted in Fig. 2 with $k_{1}=5$. At switching points, $y_{1 k}=p_{1} \sin x_{1 k}$ and $y_{2 k}=p_{2} x_{2 k} \cos x_{1 k}$. Thus, only switching points are presented in the synchronization scenario. The switching displacement and phases versus parameter $k_{2}$ for synchronization of the controlled periodic gyroscope with the chaotic gyroscope are illustrated in Fig. 2a, b. The distributions of appearance and disappearance of projective function synchronization for the master and slave systems in phase plane are presented in Fig. 2c, d, respectively. For the synchronization scenario at $k_{1}=5$, the partial projective function synchronization of two gyroscopes exists in $k_{2} \in(0.09,16.75)$. If $k_{2} \in(0,0.09)$, the projective function synchronization of chaotic motions for two systems cannot be obtained. If $k_{2} \in(16.75, \infty)$, the full, projective function synchronization of chaotic motions for such two systems is achieved. The switching scenarios are chaotic because the
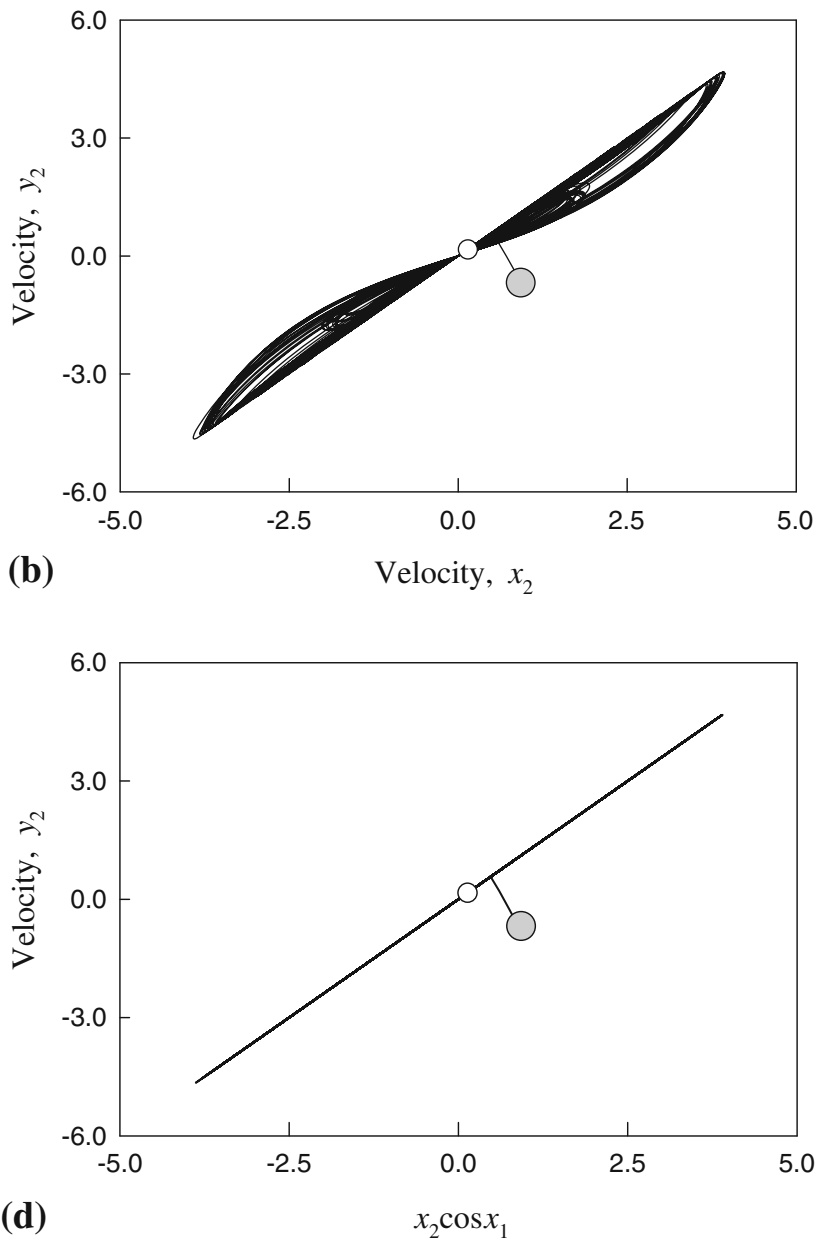

velocity $y_{2}$ versus $x_{2} \cos x_{1}$. (Hollow circular symbols are synchronization appearance, gray symbols denote the initial conditions)

motion of master system is chaotic. For a global view of projective function synchronization, the parameter map $\left(k_{1}, k_{2}\right)$ is presented in Fig. 3. Non-synchronization, partial synchronization and full synchronization are labeled by the corresponding acronyms "NS", "PS" and "FS", respectively. For $k_{1}>1.2$ and $k_{2}>16.75$, the projective function synchronization of the two systems exists. However, only the partial, projective function synchronization exist for $0<k_{1}<1.2$ and $k_{2}>16.75$. For small $k_{2}$, the non-synchronization area is given herein.

To guarantee the projective function synchronization of two gyroscope systems, the invariant domain of the slave system in Fig. 4a is generated from the conditions in Eq. (40) for the control parameters $k_{1}=5$ and $k_{2}=9$. The shaded regions are for non-synchronization, and the other areas are for synchronization. The boundaries are the maximum and minimum values for the onset and vanishing of function synchronization. For the controlled gyroscope system to synchronize with the chaotic gyroscope, the portion for the pro- 
jective function synchronization of the trajectories of two gyroscope systems should be in the synchronization invariant domain. Otherwise, the projective function synchronization for the two gyroscope systems cannot be formed. From the parameter map $\left(k_{1}, k_{2}\right)$, the partial projective function synchronization of two gyroscope systems exists for $k_{1}=5$ and $k_{2}=9$. In Fig. 4b, c, displacement and velocity responses for the master and slave gyroscope systems are partially synchronized. Hollow and filled circular symbols represent switching points for appearance and disappearance, respectively. The big gray symbols are initial conditions. In phase plane, the invariant domains of function synchronization are embedded. The projective function synchronization of trajectories lie in the invariant domain. To observe the partial function synchronization of two gyroscopes with different behaviors, the velocity $y_{2}$ versus $x_{2} \cos x_{1}$ is plotted in Fig. 4d. The time histories of displacements and G-functions are depicted in Fig. 4e, f. The symbols "N" and "S" represent "non-synchronization" and "synchronization". The non-shaded regions are for non-synchronization, and the G-functions for non-synchronization are presented by dashed curve. The shaded areas are for synchronization, and the corresponding G-functions satisfy the synchronization conditions in Eq. (38), i.e. $g_{1}<0, g_{2}>0, g_{3}<0$ and $g_{4}>0$.

From the parameter map, for $k_{1}=5$ and $k_{2}=16$, the full, projective function synchronization for the controlled periodic gyroscope with the chaotic gyroscope takes place, as shown in Figs. 5 and 6. The G-function distributions along the displacement are plotted in Fig. 5a, b. The analytical conditions for projective function synchronization in Eq. (38) is satisfied, which means the controlled periodic gyroscope is synchronized fully with the chaotic gyroscope. The phase trajectories of the master and slave systems are presented in Fig. 5c, d, and the invariant domains are also embedded. All the trajectories of two systems lie in the synchronization invariant domain. Compared to the identical synchronization, the projective function synchronization of chaotic motions cannot be observed directly because the synchronization is based on a sinusoidal function. Thus, the relationship of state variables between master and slave systems should be presented. In Fig. 6a, b, the displacement $x_{1}$ versus $y_{1}$ and the velocity $x_{2}$ versus $y_{2}$ are depicted, respectively. In addition, the displacement $y_{1}$ versus $\sin x_{1}$ and the velocity $y_{2}$ versus $x_{2} \cos x_{1}$ are also shown in Fig. $6 \mathrm{c}$, d. The state variables of two coupled gyroscopes are satisfied $y_{1}=0.5 \sin x_{1}$ and $y_{2}=1.2 x_{2} \cos x_{1}$.

\section{Conclusions}

The projective function synchronization of two gyroscopes with different motions was investigated under sinusoidal constraints. From the theory of discontinuous dynamical systems, the analytical conditions of such projective function synchronization were developed, which gives the function synchronization mechanism of two gyroscope systems. With special parameters, the partial and full projective function synchronizations were numerically simulated to illustrate analytical conditions. The corresponding invariant domains for the projective function synchronization were also obtained. This investigation provides a better understanding of the projective function synchronization dynamics of two gyroscope systems.

Acknowledgments The work is supported by the National Natural Science Foundation of China (No. 51075275), and the Ministry of Education of Overseas Returnees Start-up Research Fund, and Six Categories of Summit Talents of Jiangsu Province of China.

\section{References}

1. Pecora LM, Carroll TL (1990) Synchronization in chaotic systems. Phys Rev Lett 64:821-824

2. Rulkov NF, Sushchik MM, Tsimring LS, Abarbanel HD (1995) Generalized synchronization of chaos in directionally coupled chaotic systems. Phys Rev E 50:1642-1644

3. Maninieri R, Rehacek J (1999) Projective synchronization in threedimensional chaotic systems. Phys Rev Lett 82:3042-3045

4. Yan JJ, Hung ML, Liao TL (2006) Adaptive sliding mode control for synchronization of chaotic gyros with fully unknown parameters. J Sound Vib 298:298-306

5. Li GH (2007) Modified projective synchronization of chaotic system. Chaos Solitons Fractals 32:1786-1790

6. Hu MF, Yang YQ, Xu ZY, Guo LX (2008) Hybrid projective synchronization in a chaotic complex nonlinear system. Math Comput Simul 70:449-457

7. Hung ML, Yan JJ, Liao TL (2008) Generalized projective synchronization of chaotic nonlinear gyros coupled with dead-zone input. Chaos Solitons Fractals 35:181-187

8. Chen L, Shi YD, Wang DS (2010) Adaptive generalized synchronization between Chen system and a multi-chaotic system. Chin Phys B 19(10):100503

9. Yu YG, Li HX (2011) Adaptive hybrid projective synchronization of uncertain chaotic systems based on backstepping design. Nonlinear Anal 12:388-393

10. Wu XJ, Wang H, Lu HT (2011) Hyperchaotic secure communication via generalized function projective synchronization. Nonlinear Anal 12:1288-1299

11. Li CL (2012) Tracking control and generalized projective synchronization of a class of hyperchaotic system with unknown parameter and disturbance. Commun Nonlinear Sci Numer Simul 17:405-413

12. Wang XY, Fan B (2012) Generalized projective synchronization of a class of hyperchaotic systems based on state observer applications. Commun Nonlinear Sci Numer Simul 17:953-963

13. Niu YJ, Wang XY, Pei BN (2012) Adaptive projective synchronization of different chaotic systems with nonlinearity inputs. Chin Phys B 21(3):030503

14. Luo ACJ (2009) A theory for synchronization of dynamical systems. Commun Nonlinear Sci Numer Simul 14:1901-1951

15. Min FH, Luo ACJ (2012) On parameter characteristics of chaotic synchronization in two nonlinear gyroscope systems. Nonlinear dyn 69(3):1203-1223 
16. Min FH (2012) Analysis of generalized projective synchronization for a chaotic gyroscope with a periodic gyroscope. Commun Nonlinear Sci Numer Simul 17:4917-4929

17. Min FH, Luo ACJ (2013) Parameter characteristics of projective synchronization of two gyroscope systems with different dynamical behaviors. Discontinuity Nonlinearity Complex 2:167-182
18. Luo ACJ (2008) A theory for flow switchability in discontinuous dynamical systems. Nonlinear Anal 4:1030-1061

19. Luo ACJ (2009) Discontinuous dynamical systems on time-varying domains. Higher Education Press, Beijing

20. Luo ACJ (2013) Dynamical system synchronization. Springer, New York 